\title{
Les aménagements du bas delta de la Néra et du cordon littoral de la Roche percée (Bourail, Nouvelle-Calédonie)
}

Allenbach M. (1)*, Maître de Conférences en Géologie

Hoibian T. (2)*, Docteur en Océanologie, chercheur associé

*Laboratoire de Géosciences, Université de la Nouvelle-Calédonie.

Résumé : Le cordon littoral de la Roche percée à Bourail (Nouvelle-Calédonie) en voie d'érosion continue depuis plus de dix ans et très fortement fragilisé par le passage du cyclone Béti en 1996 a fait l'objet d'un aménagement intégré. Ce dernier a associé des travaux classiques d'enrochements dans la partie médiane de la plage à une reconstitution de l'environnement naturel au niveau de l'embouchure nord de la rivière. Le suivi du site, après passage sur zone du cyclone Frank en 1999 démontre un bon fonctionnement des ouvrages depuis leur réalisation.

Abstract: An integrated management of Roche percée offshore bar at Bourail (New-Caledonia), a place submitted to a significant erosion since more ten years has been realized after hurricane Béti spell in 1996. The management associate classic enrockment cordon in the medium part of the beach with an intervention for restoring the natural operating scheme of northern mouth of the Néra River. The topographic survey of the area, after hurricane Frank spell in 1999 shows a good functioning of the engineering works since their building.

\section{Introduction}

En mars 1996, la crue cyclonique de la rivière Néra et l'attaque de la mer liées au passage du cyclone Béti (figure 1) sur la Nouvelle-Calédonie ont entraîné en quelques heures une très forte érosion du cordon littoral de la Roche percée à Bourail, sur la côte ouest de la Grande Terre. Le site, haut lieu touristique du Territoire, déjà fragilisé par une érosion continue et étudiée (ALLENBACH M., $1998^{(1)}$ ) depuis plusieurs années a alors connu un risque majeur d'ouverture du cordon sableux urbanisé depuis une vingtaine d'années. Ce risque s'est traduit par une forte inondation du lido et la création de profonds thalwegs érosifs (figure 2) dans l'axe des écoulements de la rivière au travers du cordon. Un danger de protection civile menaçant les personnes qui vivent de façon permanente ou épisodique (ouverture brutale du cordon sableux) sur le lido a été clairement identifié, si des événements analogues à ceux connus lors du passage de Béti venaient à se reproduire sur le site.

Le Laboratoire de Géosciences a été chargé de l'étude de faisabilité d'un aménagement du bas delta de la rivière Néra. Cette étude a pris la forme d'une expertise cosignée avec le professeur R. BONNEFILLE ${ }^{(2)}$. Réalisée en mai 1997, elle a dégagé, selon les souhaits du Service du Génie Rural de la Province Sud, commanditaire de l'expertise, plusieurs variantes pour les ouvrages de génie civil côtier à édifier pour sauvegarder le site. 


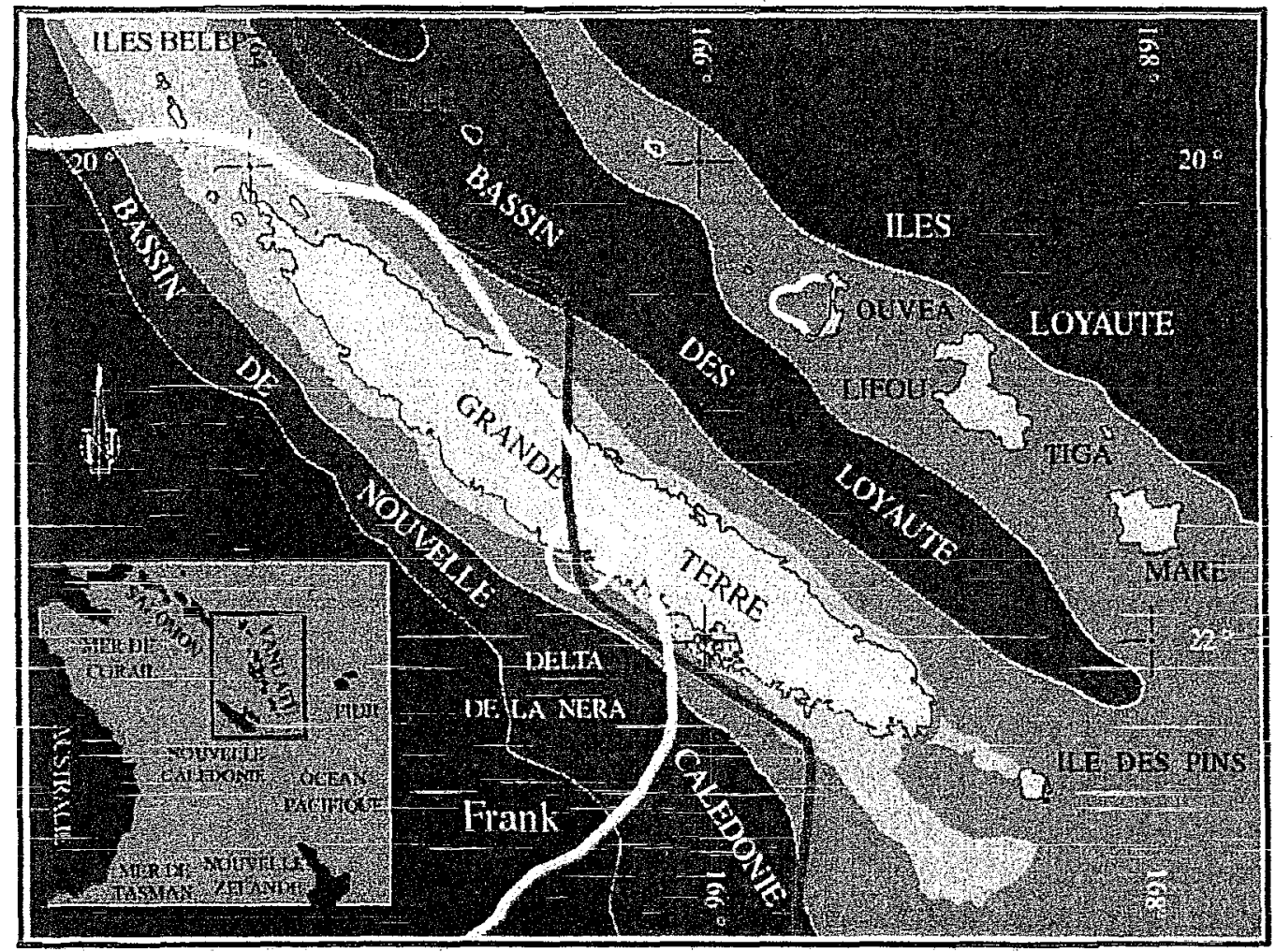

Figure 1 : Trajectoires des cyclones Béti (1996) et Frank (1999) Paths of hurricanes Beti (1996) and Frank (1999).

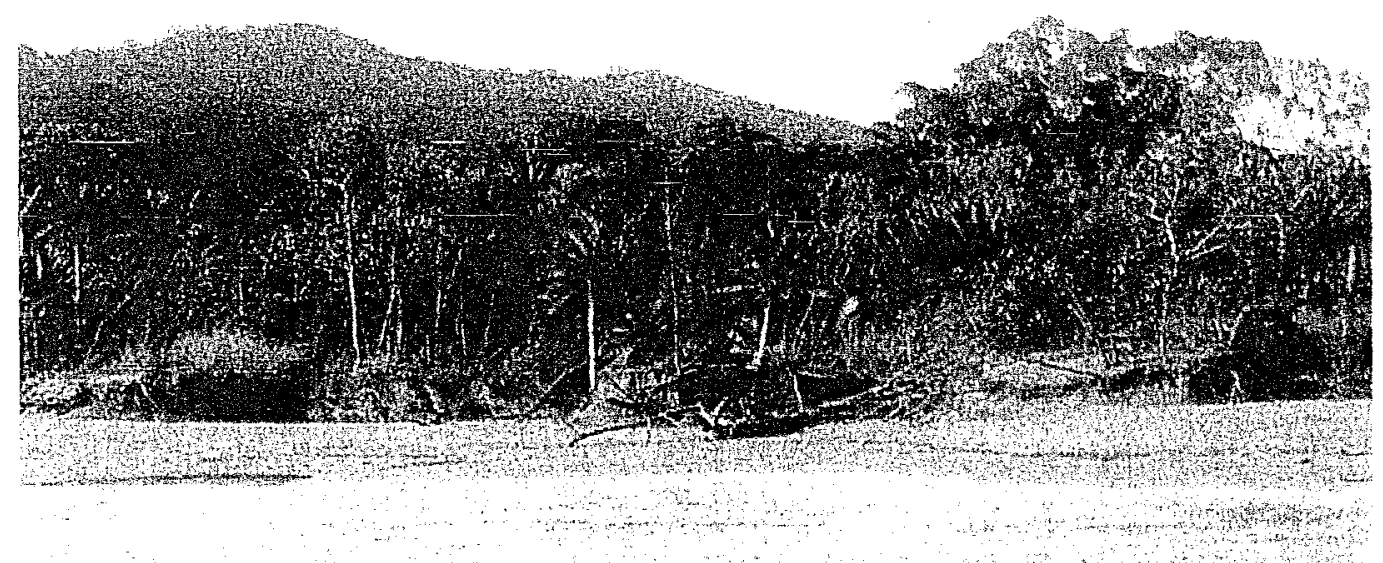

Figure 2: Erosion de la plage après le passage de Béti (1996)

Beach erosion after hurricane Beti spell (1996)

\section{Le proiet d'aménagement de l'embouchure nord}

Sur la façade maritime, au niveau de l'embouchure nord de la Néra, il a été recherché un retour vers les conditions naturelles (figure 3) qui prévalaient avant la mise en place d'une digue fusible sur le cordon destinée à pérenniser le passage des riverains vers le lido. Le principe même de la digue fusible, conçu sur une approche d'hydraulique fluviatile stricte a été abandonné. L'enrochement nord de 




Figure 3 : Vue aérienne du cordon littoral en 1993

Aerial view of the offshore bar in 1993

la digue fusible dont la présence perturbait le jeu des dérives sédimentaires devait être détruit tandis que l'enrochement sud en bordure du lido était maintenu et prolongé vers l'amont (figures 4 et 5). L'intérêt des modifications était de limiter la brèche d'ouverture dù côté urbanisé, lors des crues tout en rejetant le courant vers la falaise de la pointe du Bonhomme selon le schéma naturel de circulation des eaux de la rivière. Dans ce schéma d'aménagement, la vitesse d'écoulement du fluide est ralentie par la suppression du passage forcé à travers les cordons d'enrochements de la digue fusible mais la largeur d'ouverture du cordon est augmentée. L'aménagement devait permettre à moyen terme la réinstallation d'un cordon d'embouchure naturel, (ré-alimenté dans une phase initiale, par le stock de sable disponible en amont de la route provisoire d'accès au site) et à court terme d'arrêter l'érosion très active sur ce segment de la plage. En effet, l'édification d'une route, réalisée dans l'urgence et sans analyse de la dynamique sédimentaire en arrière du littoral pour permettre l'accès des riverains sur le cordon après le passage de Béti, avait enclenché un processus de rectification du trait de côte spectaculaire nécessitant une action de correction. L'anse formée en arrière de 


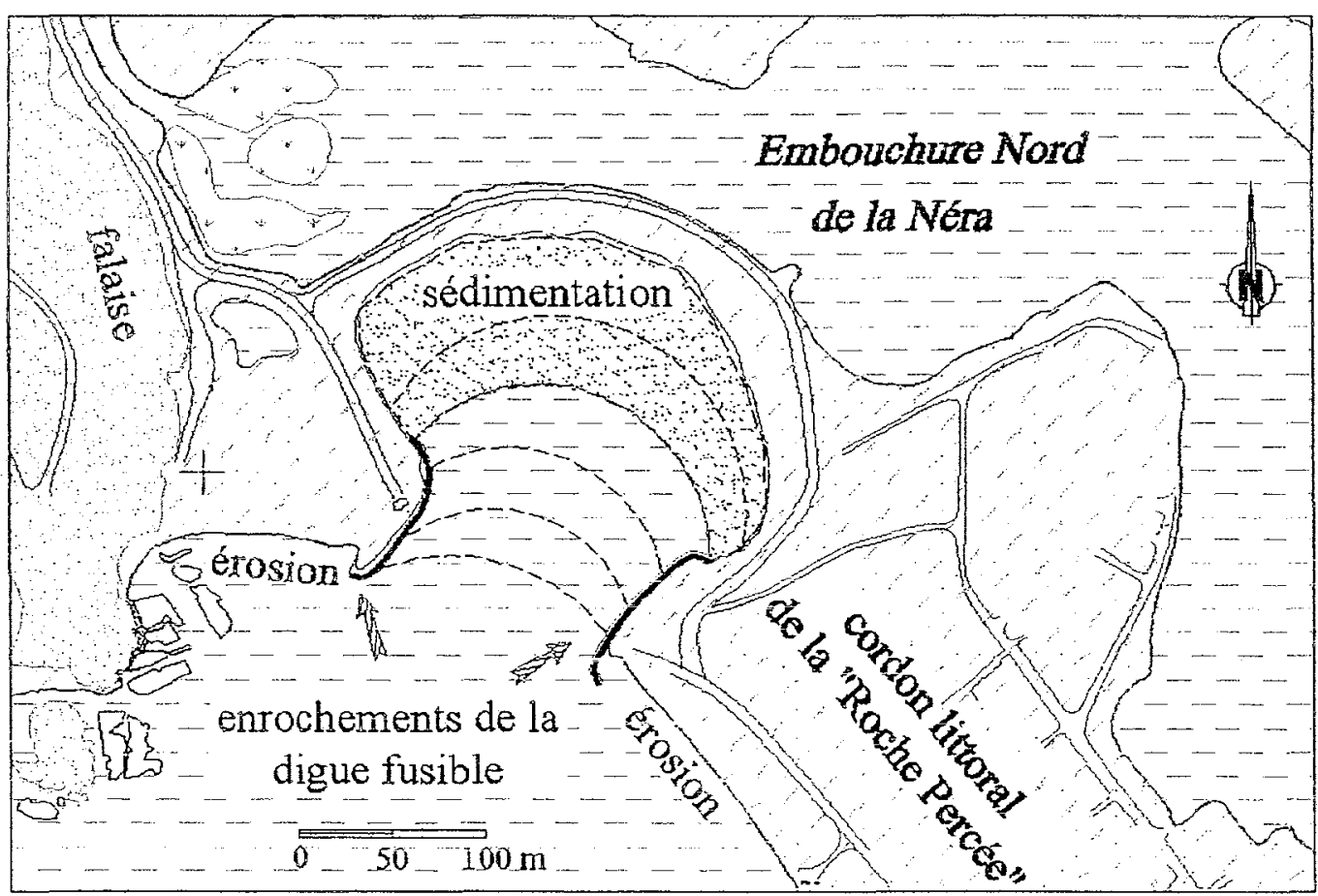

Figure 4 : Situation de l'embouchure nord avant l'aménagement. Situation of the northern mouth before engeenering works.

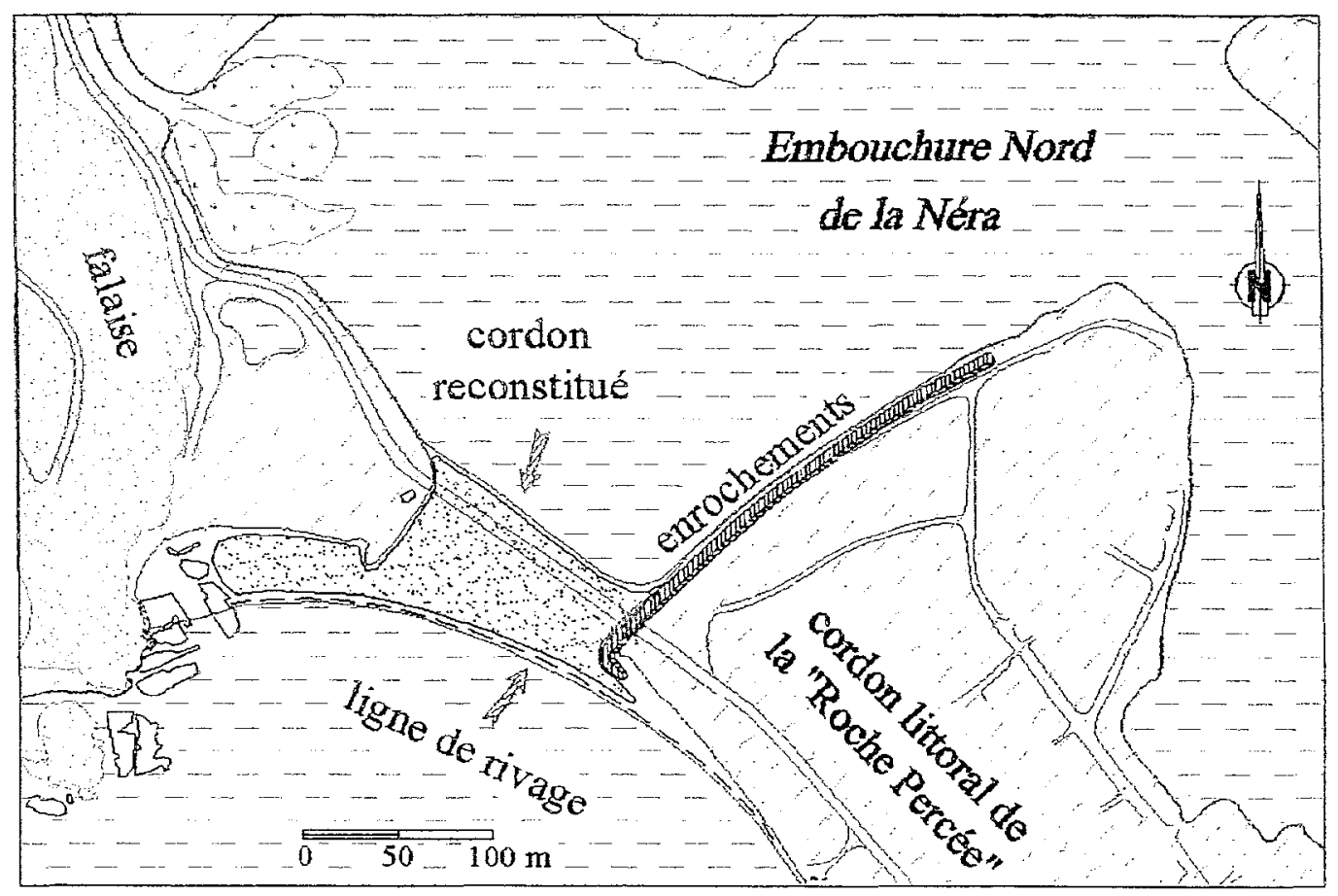

Figure 5 : Situation de l'embouchure nord après aménagement Situation of the northern mouth after engeenering works 
l'ex digue fusible s'est progressivement comblée (volume estimé à $2.10^{5} \mathrm{~m}^{3}$ ) à partir du matériel arraché à la haute plage voisine qui a reculé de près de $50 \mathrm{~m}$ en une année (figures 6 et 7 ).

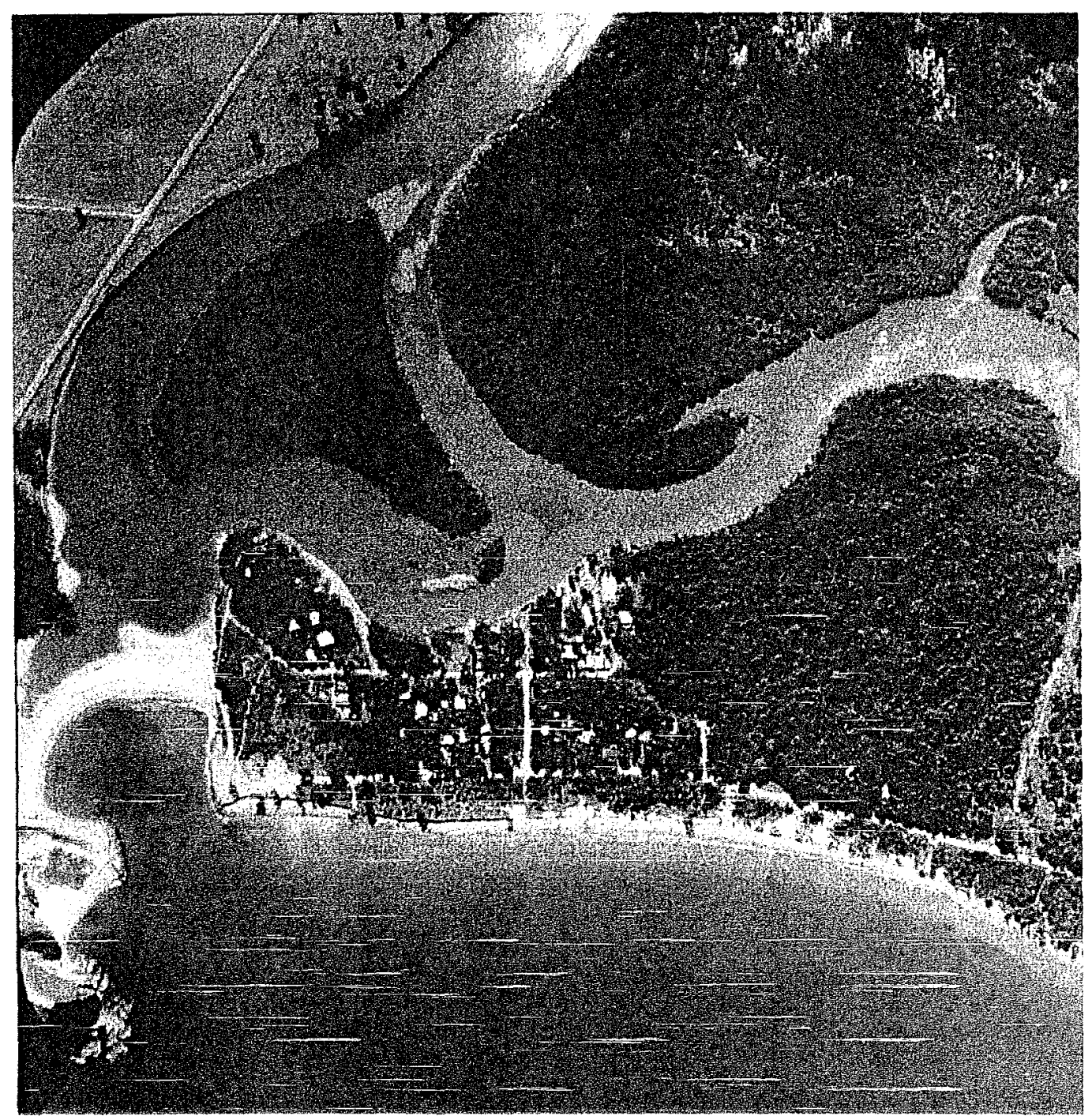

Figure 6: Vue aérienne de l'embouchure nord en juillet 1996 Aerial view of the northern mouth in july 1996

\section{Les esquisses des aménagements de la partie centrale de la plage}

Au niveau de la partie centrale de la plage, trois types de variantes ont été esquissées : brise lames, alvéoles et cordon de protection de haut de plage (figures 8 à 10). Les données naturelles des esquisses des ouvrages étaient les suivantes relevés bathymétriques détaillés, houles de périodes 10 à $12 \mathrm{~s}$ déferlant sur les ouvrages, côte maximale de la pleine mer égale à $+1,65 \mathrm{~m}$ Hydro. $(+0,80 \mathrm{~m}$ N.G.N.C.), surcôte de $1,50 \mathrm{~m}$. La grande lacune de ces données était l'absence de plans de réfraction-diffraction de la houle. Les hypothèses de travail retenues pour 




Figure 7 : Situation de l'embouchure nord en décembre 1996 Situation of northern mouth en december 1996

dessiner les coupes des ouvrages (figures 8 à 10) ont été les suivantes : masses volumiques des enrochements $=2400$ et $2600 \mathrm{~kg} / \mathrm{m}^{3} ; \mathrm{Kd}=2$ pour les musoirs et les ouvrages parallèles au rivage, $=4$ pour les parties internes des brise-lames et épis ; Pentes des talus $=3 / 1$ pour les zones où $\mathrm{Kd}=2$ et $2 / 1$ pour celles où $\mathrm{Kd}=$ 4 ; Carapaces et sous-couches comportant deux couches d'enrochements; houle de $12 \mathrm{~s}$ déferlante, de hauteur significative $\mathrm{Hs}=$ à $80 \%$ de la profondeur au pied de l'ouvrage d augmentée de $2,2 \mathrm{~m}$ pour tenir compte des surcôtes, soit $\mathrm{Hs}=4,2$ $m$ pour $d=-3 m$ N.G.N.C.

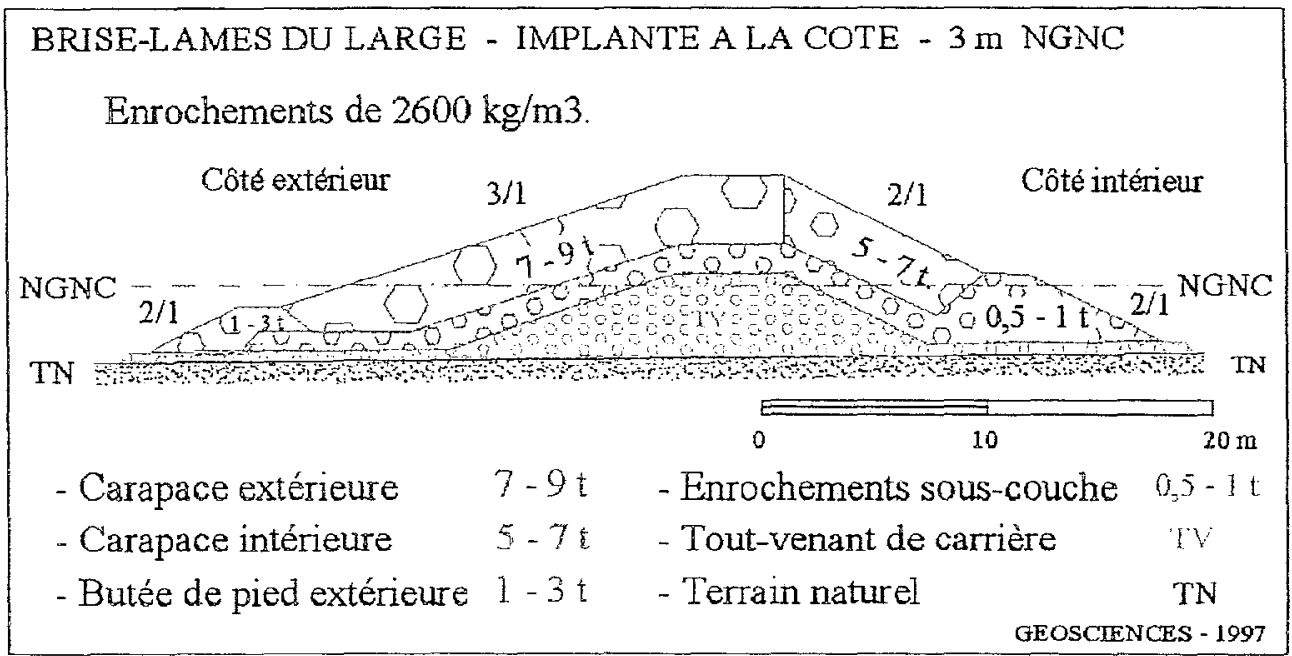

Figure 8 : Esquisse du brise lame du large.

Layout of the groyne 


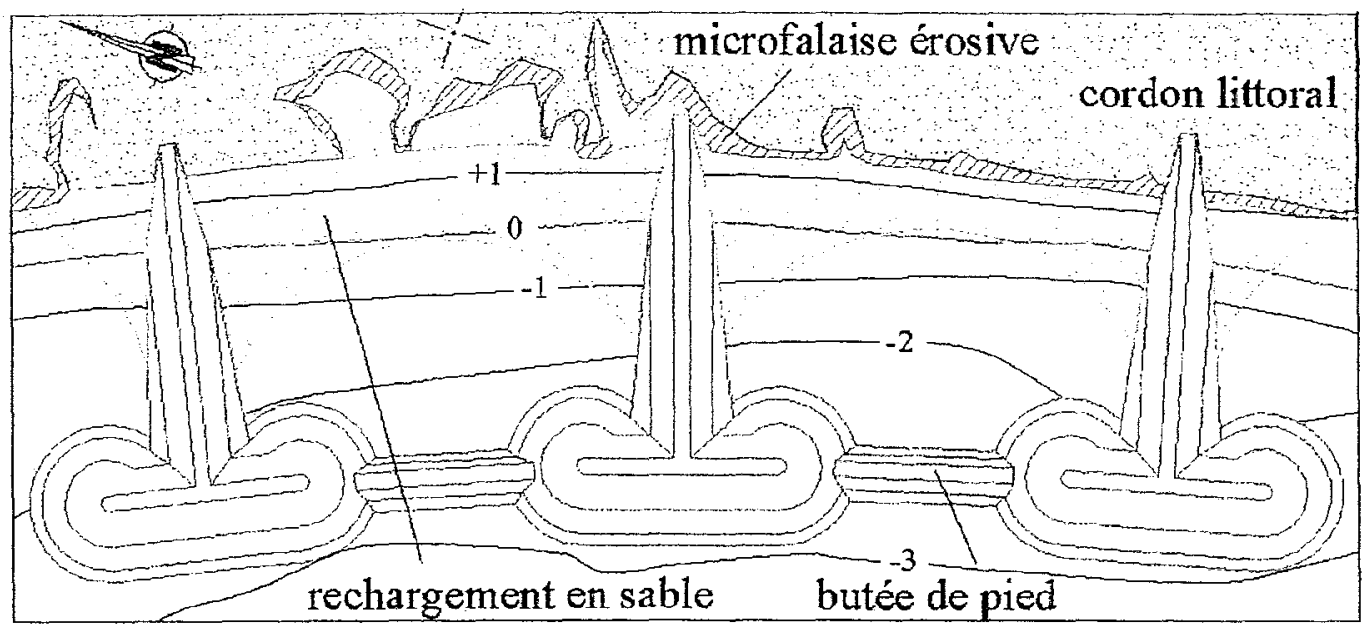

Figure 9 : Esquisse plages alvéolaires

Layout of alveolar beaches:

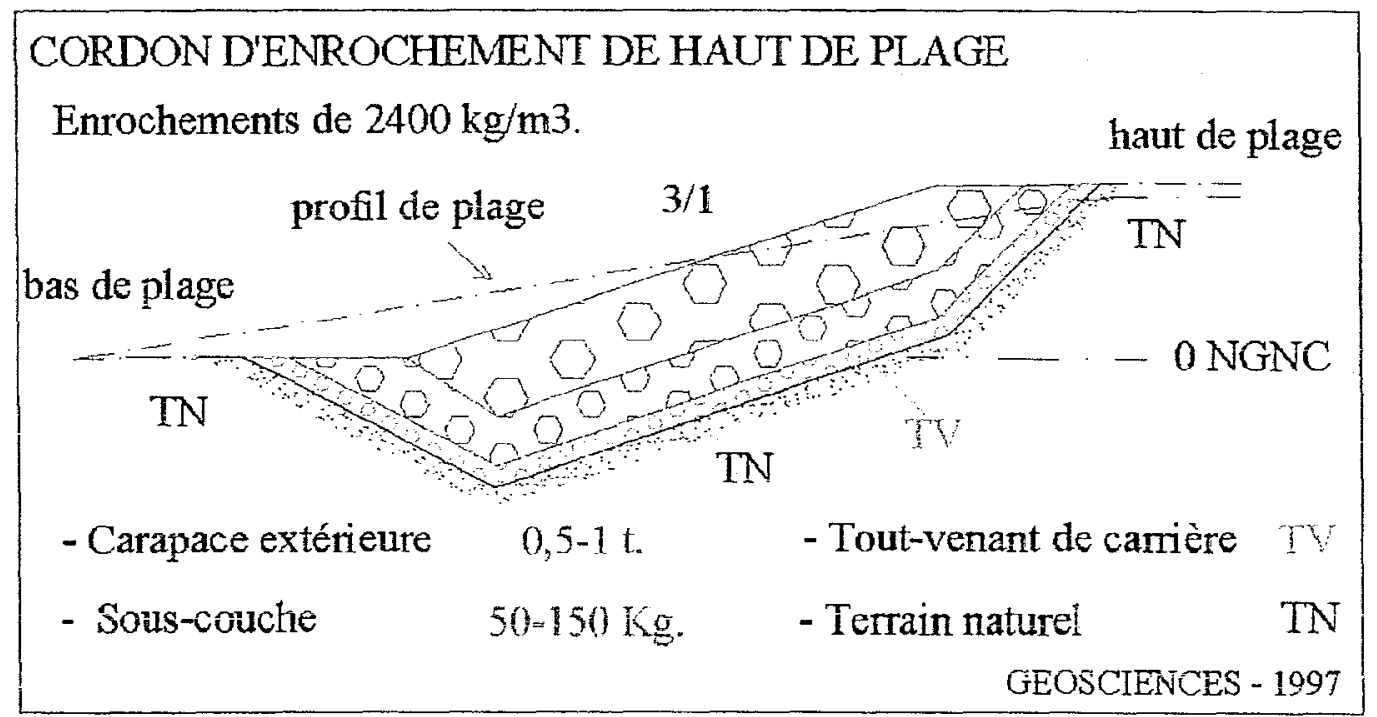

Figure 10 : esquisse cordon d'enrochement

Layout of enrockment cordon

Au niveau de la partie interne du cordon littoral, les aménagements proposaient deux axes d'actions : du confortement de berges et des actions de curage des bras de la rivière.

\section{Les travaux réalisés et leur suivi dans le temps}

Sur la base des estimatifs de coût des différentes variantes proposées, une première phase de travaux a été lancée au premier semestre 1998. Il a été réalisé côté interne du lido des actions de confortement de berges sur le linéaire le plus attaqué, c'est à dire sur la berge concave du bras de rive droite de la rivière et également en arrière du lido au droit des axes d'écoulements observés lors du passage du cyclone Béti. Sur la façade maritime, l'embouchure nord de la Néra a 
été rectifiée selon les recommandations explicitées plus avant. La solution cordon d'enrochement de haut de plage évolutive vers le système alvéolaire a été retenue pour la partie médiane de la plage et mise en place (figure 11). Le cordon arasé à la côte $+3 \mathrm{~m}$ N.G.N.C. a été édifié sur un linéaire de $350 \mathrm{~m}$ au droit de la zone fortement fragilisée en mars 1996 par le passage du cyclone Béti (cf. figure 2).

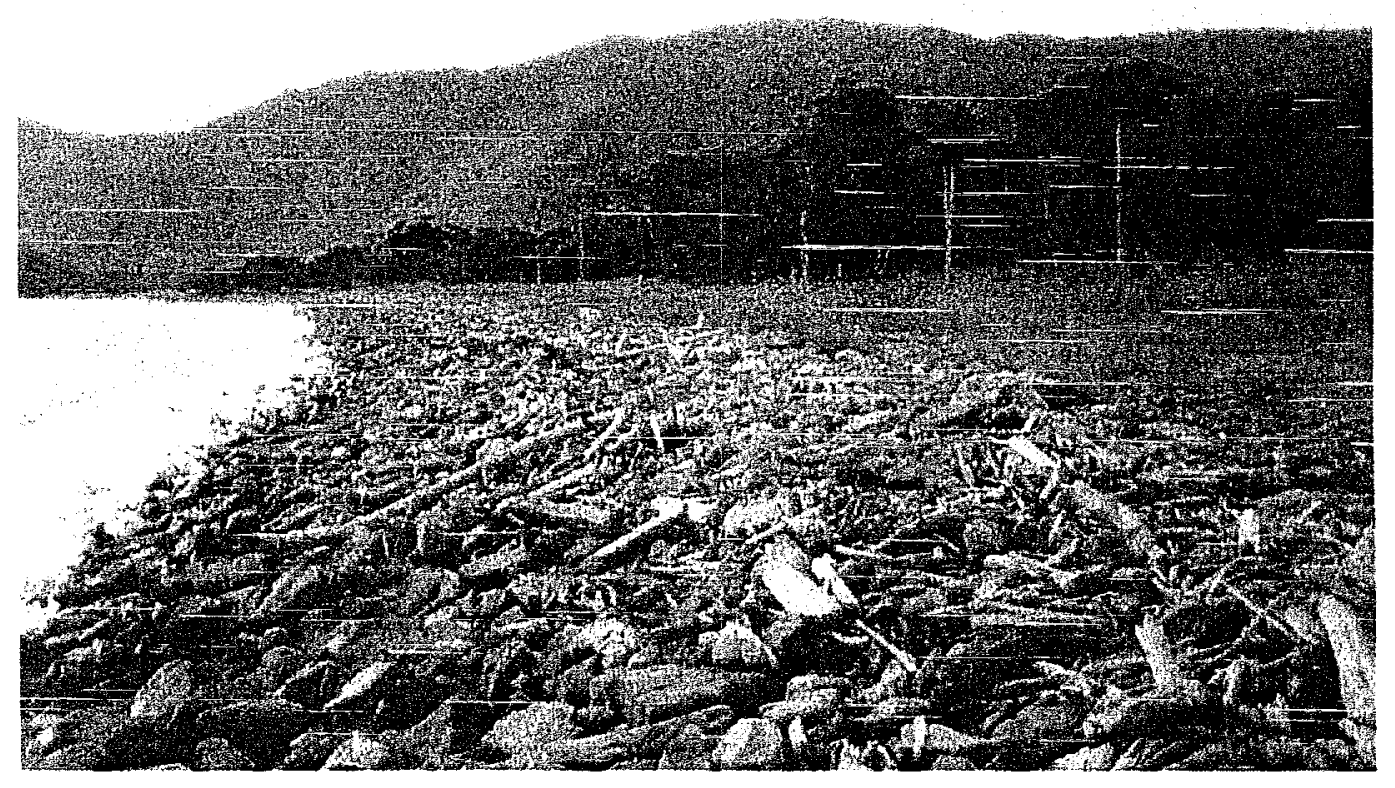

Figure 11 : Cordon d'enrochement de la partie médiane de la plage: Enrockment cordon in the medium part of the beach

Au terme de 12 mois de suivi du site post-travaux, les observations et contrôles topographiques (figures 12 et 13) montrent que l'embouchure nord est en voie de retrouver un profil d'équilibre voisin de celui prévalant avant le passage de Béti et que l'érosion est stabilisée sur la partie médiane de la plage. Dans l'axe de l'ancienne digue fusible (profil 10), le rechargement en sable effectué à partir du stock de matériaux piégés dans l'anse créée par le tracé de la route provisoire a permis dans une première phase de reconstituer une ligne de rivage évitant le phénomène d'érosion précédemment observé. Depuis cette date, l'engraissement est continu sur ce segment de plage et la situation en décembre 1999 (profil non reporté sur la figure 12) montre un gain de sédiments et une avancée de la plage sur la zone maritime. Le profil 17 (implanté dans la partie médiane de la plage, au droit du cordon d'enrochement) montre une parfaite stabilité de l'ouvrage. La zone d'arase formée de tout venant et de sable et correspondant au remblaiement des thalwegs érosifs creusés par Béti est en voie de recolonisation par la végétation naturelle (cf. figure 11), traduisant ainsi un retour à la stabilité de la zone. 


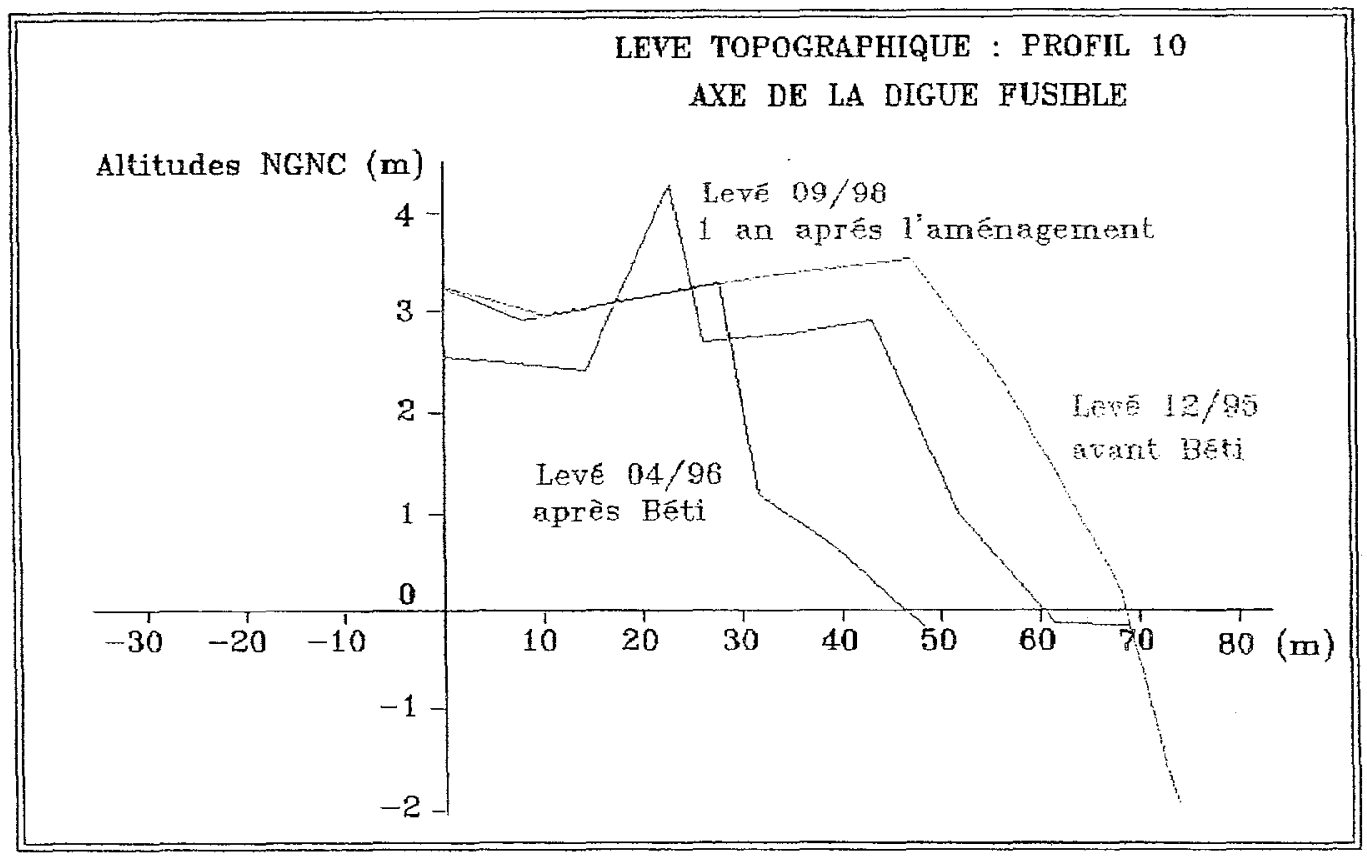

Figure $11:$ Evolution du profil de plage au droit de l'embouchure nord Evolution of beach profil in the axis of the northern mouth

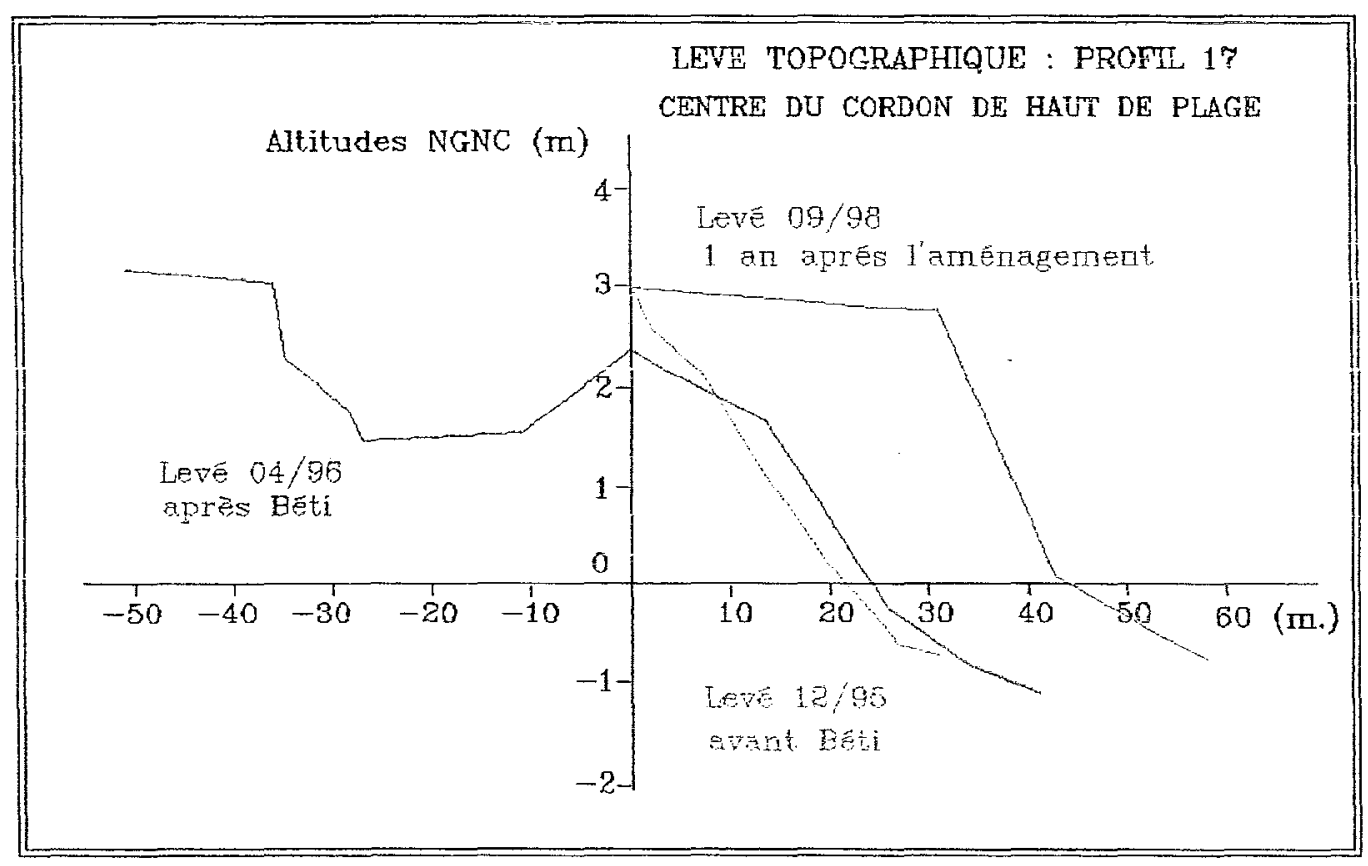

Figure 12 : Evolution du profil de plage au droit du cordon d'enrochement Evolution of beach profile in the axis of the enrockment cordon 
Le passage sur le site du cyclone Frank en février 1999, de caractéristiques très voisines de celles de Béti (cf. figure 1 et tableau 1) n'a pas entraîné de destruction sur le cordon littoral de la Roche percée.

\begin{tabular}{|lll|}
\hline Cyclone & BETI & FRANK \\
Date passage sur Bourail & $27 / 03 / 96$ & $20 / 02 / 99$ \\
Précipitation journalière maximale & $175,8 \mathrm{~mm}$ & $197,5 \mathrm{~mm}$ \\
Vent instantané maximum & $151 \mathrm{~km} / \mathrm{h}$ & $140 \mathrm{~km} / \mathrm{h}$ \\
\hline
\end{tabular}

Tableau 1 : Comparatif entre les caractéristiques des cyclones Béti et Frank. Comparison between charateristics of hurricanes Béti et Frank.

L'évacuation de la crue cyclonique a été facilitée par l'ouverture précoce du cordon, calé à une altitude inférieure à celle de la digue fusible $(2,5 \mathrm{~m}$ N.G.N.C aujourd'hui contre $3 \mathrm{~m}$ N.G.N.C.). L'ouverture du cordon s'est effectuée sur l'ensemble du linéaire côtier compris entre le cordon d'enrochement mis en place en bordure nord du lido et la falaise de flysch du Bonhomme. Ce faisant, le lit sableux de la rivière n'a pas été profondément entaillé par le courant d'évacuation de la crue. La profondeur maximale de la zone d'ouverture a été mesurée à $-2 \mathrm{~m}$, contre $-8 \mathrm{~m}$, en mars 1996 où le sable avait été entièrement enlevé et la roche sous-jacente mise à nue. La reconstruction de la route sur le cordon d'embouchure faiblement déstructuré par la crue a pu être rapidement réalisée par des moyens légers. L'inondation du lido, classiquement observée lors de chaque crue importante de la Néra a été fortement minimisée par l'ouverture précoce du cordon naturel, contrairement aux faits observés lors de Béti. Pour cet épisode cyclonique, le matériel accumulé en arrière de la digue (charge solide du bras de rive droite de la Néra) au cours des cinq années s'étant écoulées entre la construction de l'ouvrage et son ouverture en mars 1996 s'était opposé à l'ouverture de l'ouvrage. L'effet fusible escompté avait été inopérant en début de crue et avait engendré les écoulements au travers du lido par effet de débordement du lit de rive droite, jusqu'à la rupture tardive de l'ouvrage. Cet effet de débordement n'a pas été observé en Février 1999. Aucun dommage n’a été noté au niveau du cordon d'enrochements mis en place sur la partie centrale de la plage et en arrière de celui-ci. Partant de ce constat du bon fonctionnement de l'aménagement réalisé, les décideurs économiques et politiques du Territoire ont fait le choix de ne pas aller, pour l'instant, au delà des travaux déjà entrepris et l'édification des alvéoles est différée.

(1) ALLENBACH M. (1998) - L'érosion du cordon littoral de la Roche percée (Bourail, Nouvelle-Calédonie). Dynamique et commandes du processus érosif. C.R. Vèmes Journées nationales génie civil - génie côtier, Toulon, 13-15 mai, Session IV, pp 321-328.

${ }^{(2)}$ M. ALLENBACH, R. BONNEFILLE, (1997). Expertise sur la faisabilité d'un aménagement intégré du delta de la Néra. Rapport de la Convention de recherche UFP/Province Sud de Nouvelle-Calédonie, $50 \mathrm{p}$. 